\section{Macrophages get a CAR}

Despite the efficacy of chimeric antigen receptor (CAR) T cell therapy in treating haematological malignancies, its application in solid tumours has proved challenging. This is partly due to the fact that $\mathrm{T}$ cells cannot easily find, penetrate and survive in the tumour microenvironment (TME). To overcome these challenges, researchers have explored other immune cell types as CAR platforms, which might circumvent some of the limitations of CAR T cells. Klichinsky et al. have engineered macrophages to express CARs that target their phagocytic activity towards tumour cells. These macrophages (CAR-Ms) reduced the tumour burden and prolonged overall survival in mice.

As central effectors of the innate immune response, macrophages detect and eliminate abnormal and infected cells. Macrophages are also abundant within the TME and therefore could be particularly well suited to trafficking to and surviving within it. The authors wondered whether the expression of CARs in macrophages could redirect their phagocytic function to tumour cells while stimulating an adaptive immune response. "When Mike [Klichinsky] and I started working on this project in 2014, we pursued it as a pure intellectual exercise: what would it look like if you could put CARs on macrophages, and where would this technology best fit if successful?" explains Saar Gill, lead author of the study. "We subsequently discovered that in the 1990s and early 2000s there were several clinical trials of non-engineered macrophage infusions for patients with metastatic solid cancers," he says. These studies, in which high numbers of autologous monocyte-derived macrophages were expanded and administered to patients, proved that the approach was safe. However, the trials did not show antitumour efficacy. Redirecting the phagocytic function of macrophages and stimulating the adaptive immune system with a CAR could change this outcome, Gill reasoned.
First, the authors transduced a human macrophage cell line with a first-generation anti-CD19 CAR gene that encodes the $\mathrm{CD} 3 \zeta$ intracellular domain. As macrophages are part of the first line of defence against viral infection, genetically engineering these cell lines was a major hurdle. "We tested multiple viral vectors including lentiviral, retroviral and multiple adenoassociated virus serotypes but found that human macrophages are innately resistant to infection by these," Gill explains. "It was serendipitous that we found a chimeric adenoviral vector called Ad5F35 (created by others) that could mediate gene transfer into human macrophages with high efficiency, leading to high and sustained expression of the CAR." These CAR-Ms - as well as CAR-Ms subsequently generated against solid tumour cells expressing mesothelin or HER2 - phagocytosed the antigen-positive cells that they were designed to target.

The authors next tested the antitumour activity of CAR-Ms in vivo in different models of immunodeficient mice with diminished macrophage function. Mice injected with SKOV3 a HER $2^{+}$ovarian cancer cell line - and treated with a single dose of HER2-targeted CAR-Ms (intravenously or intraperitoneally) showed a substantial reduction in tumour burden and prolonged survival, although all mice eventually progressed. In xenograft models of five different types of solid tumour, CAR-Ms trafficked to all five types.

Importantly, the authors observed that transduction with Ad5F35 vector - regardless of CAR expression - induced a pro-inflammatory M1 phenotype in the infected macrophages with upregulation of interferon-induced and antigen-presenting machinery genes, as opposed to the immunosuppressive and pro-tumorigenic M2 phenotype found in tumour-associated macrophages.

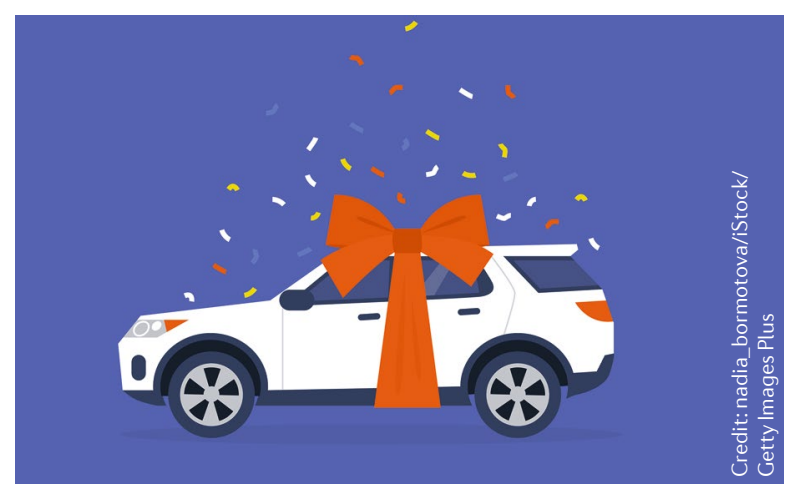

16

the expression

of CARs in macrophages could redirect their phagocytic function to tumour cells
In vitro, co-culture experiments showed that CAR-Ms induced a pro-inflammatory phenotype in M2 macrophages, activation and maturation of dendritic cells, and recruitment of resting and activated T cells. In vivo, mice with metastatic SKOV3-derived xenografts treated with HER2-targeted CAR-Ms and donor-derived polyclonal (non-specific) T cells showed a better antitumour response than mice treated with CAR-Ms or T cells alone. This synergy is probably due to CAR-M-induced potentiation of T cell antitumour activity. Further experiments showed that CAR-Ms cross-presented intracellular tumour-derived antigens from phagocytosed cells.

The biotech company founded by Gill and the team (Carisma Therapeutics) is ready to initiate a phase I trial to assess CAR-Ms in patients with metastatic HER2-overexpressing tumours. "Not just breast cancer: many other epithelial malignancies also overexpress HER2 yet do not respond to the commonly used monoclonal antibody Herceptin or its derivatives," says Gill.

These results highlight the role of the innate immune system when considering immunotherapeutic approaches and add another platform to the increasing number of cell therapy modalities to treat solid tumours.

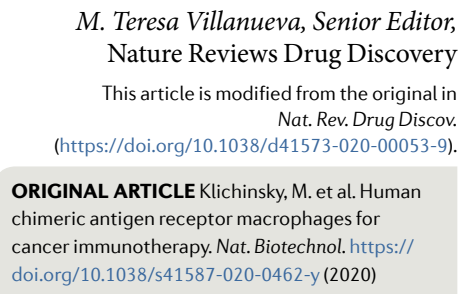

M. Teresa Villanueva, Senior Editor, Nature Reviews Drug Discovery This article is modified from the original in Nat. Rev. Drug Discov. (https://doi.org/10.1038/d41573-020-00053-9). ORIGINAL ARTICLE Klichinsky, M. et al. Human chimeric antigen receptor macrophages for cancer immunotherapy. Nat. Biotechnol. https:// doi.org/10.1038/s41587-020-0462-y (2020) 